\title{
STELLAR CONVECTIVE CORES
}

\author{
I.W. ROXBURGH \\ Astronomy Unit, Queen Mary College, University of London \\ Mile End Road, London E1 4NS, UK
}

\section{Abstract and Introduction}

The internal structure of stars is governed by hydrostatic support, the distribution of the chemical elements, the transport of energy by radiation and convection, and the liberation of energy by nuclear reactions. The evolution of stars is primarily determined by the changing composition due to the nuclear burning of elements in the central parts of the star, and the redistribution of the products of these reactions by mixing processes. The dominant mixing process is convection: it governs the extent of the mixed cores in moderate and large mass main sequence stars and their subsequent evolution, it mixes nuclear processed material into the envelopes of giants affecting the composition of material ejected into the interstellar medium, thereby affecting the chemical (and luminosity) evolution of galaxies. Understanding convection is essential if one is to understand the evolution of stars. Here I am concerned with convection in stellar cores and in particular with the extension of these cores by the penetration of convective motions into the surrounding stable layers affecting the internal structure and enlarging the chemically mixed region, which in turn affects the subsequent evolution. I briefly discuss a number of approaches to this problem: isochrone fitting of clusters and binary stars; simple theoretical models, the integral constraint, numerical simulation and what we can hope to get from asteroseismological observations of individual stars and of clusters and stellar groups.

\section{Isochrone fitting to binary stars and stellar clusters}

Since the structure and evolution of a star is affected by the extent of its convective core so too will be the isochrone (or locus at constant age) of a group of stars in the $\mathrm{H}-\mathrm{R}$ diagram. One way to seek to quantify overshooting is therefore to seek the best fit to both the H-R diagram of individual clusters and the width of the main sequence band for a collection of clusters with differnt assumptions on convective overshooting.

In studies by Maeder and Mermillod (1992) and Meynet et al. (1993), overshooting was parametrised by taking the radius of core to be enlarged by a multiple $\alpha$ of the local pressure scale height $H_{p}=P /|d P / d r|$. These authors found that the best fit to isochrones of a large number of clusters indicated that $\alpha \approx 0.2-0.3 H_{p}$. In a more recent study Dowler and VandenBerg (1995, private communication) found that 
cluster isochrones could be well fitted if core overshooting was as given by the integral condition I derived some years ago (Roxburgh 1978,89, see section 5). Figure 1 shows their results for the Cluster IC 4651. Similar results were found for the range of clusters they analysed. These authors also found that the same model of core overshooting improved the fit of theoretical evolutionary models with the observations of binary stars.

\section{The classical mixing length model of convection}

The usual approach to modelling convection is to use the mixing length model, or some presumed refinement thereof, but at best such models only give an indication of the magnitude of the effects being studied, and their quantitative predictions should not be taken seriously. Unfortunately not all astronomers exercise such restraint! In this "classical" mixing length model of convection, turbulent eddies are imagined to rise or sink under the action of bouyancy, travelling a distance $\ell$, the mixing length, conserving entropy and in pressure equilibrium with their surroundings, and then to mix into their surroundings. The mixing length $\ell$ is taken as $\alpha H_{p}$ where $H_{p}$ is the pressure scale height and $\alpha$ is an unknown parameter adjusted so that a solar model has the observed solar radius, or by some other empirical fitting condition.

In this simple model the temperature excess $\delta T$, density excess $\delta \rho$, velocity $v$ and fluxes $F_{\text {rad }}, F_{\text {conv }}$ are given by

$$
\begin{gathered}
\frac{d}{d z}\left(\frac{\delta T}{T}\right)=\Delta \nabla \frac{1}{H_{p}}, \quad v \frac{d v}{d z}=g \frac{\delta T}{T}, \quad \Delta \nabla=\nabla-\nabla_{a d}, \quad \nabla=\frac{d \log T}{d \log P} \\
F_{r a d}=\chi\left(\nabla_{a d}+\Delta \nabla\right), \quad F_{c o n v}=c_{p} \rho T v\left(\frac{\delta T}{T}\right), \quad F_{r a d}+F_{c o n v}=F, \quad \chi=\frac{4 a c T^{4}}{3 \kappa \rho H_{p}}
\end{gathered}
$$

where $\nabla_{a d}=(\Gamma-1) / \Gamma$ with $\Gamma=c_{p} / c_{v}$ the ratio of specific heats. The kinetic energy flux is neglected on the (weak) argument that upward and downward moving eddies occupy equal areas and the viscous energy flux is asssumed negligibly small. Taking $\Delta \nabla, g, H_{p}, \rho, T$ constant and integrating over the mixing length $\ell=\alpha H_{p}$ gives

$$
\delta T \approx \alpha T \Delta \nabla, \quad v^{2} \approx \alpha^{2} c_{p} T \Delta \nabla, \quad F_{c o n v} \approx \alpha^{2} \rho\left(c_{p} T\right)^{3 / 2}(\Delta \nabla)^{3 / 2} .
$$

Deep inside the convective zone with $F_{\text {conv }} \approx F, \Delta \nabla \approx 10^{-8}$. At the boundary $(z=0), v=0, \Delta \nabla=0, F_{\text {rad }}=F=\chi_{0} \nabla_{a d}, F_{c o n v}=0$. On the unstable side of the boundary $(z<0), F_{\text {rad }} \approx(1-x) F, F_{\text {conv }} \approx x F, \Delta \nabla \approx x^{2 / 3} \Delta \nabla_{0}$ where $x=-z / H_{\chi}$ and $\Delta \nabla_{0}$ is the value well inside the stable layer In the stable region $F_{\text {conv }}=$ $0, F_{\text {rad }}=F=\left(\chi_{0}+\chi^{\prime} z\right)\left(\nabla_{a d}+\Delta \nabla\right)$ and $\Delta \nabla \approx-\left(z / H_{\chi}\right) \nabla_{a d}$ decreases to a value of about $-\nabla_{a d} / 2$ in a distance of order $H_{\chi} / 2$. There is no convective penetration into the surrounding stable region in this simple model.

However since $\Delta \nabla \geq 0$ in the unstable region eddies are accelerated up to the boundary and therefore continue into the stable layers. With $\Delta \nabla \approx\left(-z / H_{\chi}\right)^{2 / 3} \Delta \nabla_{0}$ an eddy starting from $z=-h$ with $v=0, \delta T=0$ accelerates under gravity and arrives at the boundary $z=0$ with 


$$
v_{s}^{2}=2 \int_{-h}^{0} g \frac{\delta T}{T} d z=\frac{3}{4}\left(\frac{g \Delta \nabla_{0}}{H_{p} H_{\chi}^{2 / 3}}\right) h^{8 / 3}, \quad \frac{\delta T_{s}}{T}=\frac{3}{5}\left(\frac{\Delta \nabla_{0}}{H_{p} H_{\chi}^{2 / 3}}\right) h^{5 / 3} .
$$

The eddy continues into the stable layer where $\Delta \nabla \approx-\left(z / H_{\chi}\right) \nabla_{a d}$ and for $z>0$ has a temperature excess $\delta T$ and velocity $v$ given by

$$
\frac{\delta T}{T}=\frac{3}{5} \Delta \nabla_{0}-\frac{1}{2} \nabla_{a d} x^{2}, \quad \frac{v^{2}}{v_{0}^{2}}=\frac{3}{8}+\frac{3}{5} x-\left(\frac{1}{6} \frac{\nabla_{a d}}{\Delta \nabla_{0}}\right) x^{3}
$$

where $v_{0}^{2}=0.8 c_{p} T \Delta \nabla_{0}, x=z / H_{p}$ and we have taken $H_{\chi}=H_{p}$. The penetration distance $d$ is given by $v=0$ which with $\Delta \nabla_{0} \approx 10^{-8}$ gives $x=d / H_{p} \approx 0.004$, a very small distance.

This calculation is essentially the same as that in Roxburgh (1965), which was likewise based on an eddy picture, and closely related to that of Saslaw and Schwarzschild (1965) in which they calculated the eigenfunction of the lowest unstable eigenmode. In both cases the variation of $\Delta \nabla$ was taken as given by a model with no overshooting as in the above analysis.

\section{Non-local models of convective overshooting}

The error in these analyses was to ignore the feedback of the overshooting on the thermal structure. Even a small amount of penetration disturbs the structure of the thin overshooting region, mixing matter from the convective layer and therefore sharing entropy and making the layer slightly less stable. With a slightly less stable layer the next eddy or convective cell can penetrate that little bit further, increasing slightly the region that is mixed with the unstable layer. Since the convective turnover times are very short compared with the thermal relaxation time of a star these successive mixings change the entropy gradient in the overshoot layer making it almost adiabatic. It is necessary therefore to determine the equilibrium structure after many such mixings.

A number of non-local models have been developed to seek to incorporate this feedback; Shaviv and Salpeter (1973), Maeder (1975), Roxburgh (1978, 1985), Schmitt et al. (1984) and Zahn (1991). Whilst these models incorporate some measure of feed back they still consider convection as an extended local phenomenon. It is the authors view that since the "mixing time" in a convective region is small compared with the thermal relaxation time the mean equilibrium structure of a convecting region is a global rather than a local property. Many years ago I developed (but never published) a global model in which the structure of a convective core and surrounding layers was determined by the condition that the mean convective flux, calculated with the properties of the lowest unstable eigenmode, exactly balanced the difference between the total and radiative fluxes (cf. Roxburgh 1994). This model gave similar results to the integral constraint on convective overshooting discussed in section 5 , not surprisingly since these models, and indeed the non-local models referred to above, all neglect viscous dissipation.

In a non-local model we take the boundary of the classical convective core to be at the place where $F_{\text {rad }}=F$. Neglecting the kinetic energy flux this requires $F_{\text {conv }}$ to be zero so we consider eddies arriving at the boundary $z=0$ with $\delta T=0, v_{0}>0$. For 
simplicity we also take $\Delta \nabla=0$ at the boundary but there is no difficulty in extending the analysis to non zero values.

The equilibrium of the overshoot region is given by exactly the same equations (1), (2), as the standard mixing length model. These equations can be integrated (numerically) to give $v(z), \delta T(z), \Delta \nabla(z)$ in the overshoot region given $\delta T(0)=0, v(0), \Delta \nabla(0)$ at $z=0$ Since $d v^{2} / d z=0$ at $z=0$ and $v(z)$ is a slowly varying function of $z$, with $\Delta \nabla(0)=0$, equations (1) and (2) can be integrated to give

$$
\Delta \nabla(x) \approx-\frac{\nabla_{a d}}{1+\Lambda}\left[1-e^{-(1+\Lambda) x}\right], \quad \text { where } \Lambda=\frac{c_{p} \rho T v \nabla_{a d}}{F}, \quad x=z / H_{\chi}
$$

$\Delta \nabla$ remains small in the overshoot region as long as $\Lambda>1$ or $v>v_{0} \Delta \nabla_{0} / \nabla_{a d}$ where $v_{0}, \Delta \nabla_{0}$, are typical values inside the convectively unstable region. The variation of $v$ and the penetration distance $d$ where $v=0$ can be estimated by multiplying the second of equations (1) by $v$, using $F_{c o n v}=c_{p} \rho T v \delta T$ to eliminate $v \delta T$, noting that $F_{\text {conv }}=F-F_{\text {rad }} \approx-F z / H_{\chi}$ and integrating to give (cf. Zahn 1991)

$$
v^{3}=v_{0}^{3}-\frac{3}{5} \frac{F}{\rho} \frac{z^{2}}{H_{\chi} H_{p}}=0 \text { when } d=\left(\frac{5 \rho v_{0}^{3}}{3 F}\right)^{1 / 2}\left(H_{\chi} H_{p}\right)^{1 / 2}
$$

These results should not be taken too literally, but they show that inclusion of the feedback of the convective overshooting on the thermal structure gives a large slightly subadiabatic region out to a distance of the order of (say) 0.3 of a scale height. The mass of the convectively mixed region is then substantially greater than that of the unstable convective core, with considerable consequences for the structure and evolution of the star.

\section{The Integral Constraint on convective overshooting}

An alternative approach is to seek analytical constraints on the maximum extent of convective overshooting (Roxburgh 1978,89,92). On using the equation of continuity $\partial \rho / \partial t+\operatorname{div}(\rho \mathbf{v})=0$ the thermal energy equation $(d S=d Q / T)$ can be expressed as:

$$
\frac{\partial}{\partial t}(\rho S)+\operatorname{div}(\rho S \mathbf{v})=\frac{\Phi+\epsilon \rho-\operatorname{div} \mathbf{F}_{r}}{T}
$$

where $S$ is the entropy per unit mass, $\rho$ the density, $\mathbf{v}$ the velocity, $\mathbf{F}_{\mathbf{r}}$ the radiative flux, $\epsilon$ the energy generation per unit mass and $\Phi$ the viscous dissipation per unit volume.

On integrating this equation over a volume $V$ containing both the convective core and any overshoot region such that the convective velocites $\mathbf{v}=0$ outside $V$ then on using Gauss's theorem this can be expressed as

$$
\frac{\partial}{\partial t} \int_{V} \rho S d V+\int_{\Sigma} \rho S \mathbf{v} \cdot d \boldsymbol{\Sigma}=\int_{V} \frac{\left(\Phi+\epsilon \rho-\operatorname{div} \mathbf{F}_{r}\right)}{T} d V
$$

where $\Sigma$ is the surface of $V$. For a stationary or statistically steady state the first term on the left hand side is zero, and with $\mathbf{v}=0$ on $\Sigma$ the second term also vanishes so that the right hand side is zero. The total flux $\mathbf{F}$ is given in terms of the energy generation by $\operatorname{div} \mathbf{F}=\epsilon \rho$, and on integrating by parts, using Gauss's theorem to convert to a surface integral, and noting that $\mathbf{F}_{\mathbf{r}}=\mathbf{F}$ on $\Sigma$, we find 


$$
\int_{V}\left(\mathbf{F}_{r}-\mathbf{F}\right) \cdot \nabla\left(\frac{1}{T}\right) d V=\int_{V} \frac{\Phi}{T} d V>0
$$

where the last inequality comes from the fact that the viscous dissipation $\Phi$ is positive definite. If the viscous dissipation is neglected, (as is done in the simple non-local models described above) then since $F_{r}<F$ inside the unstable layer it follows that there must be a penetration region where $F_{r}>F$ the integral condition allowing one to determine the extent of this region, quantifying the earlier estimates derived using the eddy/plume mixing length models. But there is a major conceptual difference between the integral condition and other analyses which, in the author's opinion, is of major importance. The integral condition is a global condition on the whole convective region not just a condition in the neighbourhood of the boundary, that is the whole convecting region has to adjust to satisfy this constraint (including viscous dissipation). This is entirely reasonable since we impose the condition of statistical stationarity.

It should be stressed that the viscous dissipation is not zero, indeed is necessarily positive. In Roxburgh (1978) $\Phi$ was set zero to give an estimate of the upper limit on the extent of convective penetration, in contrast to the classical model where the kinetic energy flux is set to zero and there is no overshooting. Simple stellar models using this condition give an enhancement of the core mass of the order of $50 \%$ (Roxburgh 1978). It is this condition (with $\Phi$ neglected) that was used in the work of Dowler and VandenBerg described in section 2 and Figure 1.

However 2-D and 3-D resolved numerical simulations of convection in an unstable layer surrounded by stable layers (Roxburgh and Simmons 1993, Roxburgh 1998) demonstrated that, within their assumptions, the contribution of viscous dissipation to the integral condition decreased as the Prandtl number was decreased, and for Prandtl numbers less than 0.1 the mean properties of the solution were adequately described by the integral condition with $\Phi=0$ (Figure 2).

\section{Numerical simulation of convection}

There are several groups undertaking 2-dimensional and 3-dimensional numerical simulation of convection in the sun and stars, mostly for plane parallel geometries rather than for convective cores. I am currently developing my 3-D code for studing overshooting from cores but unfortunately this has not yet been completed. Whilst such simulations can advance our understanding of convection it is important to remember that they do not simulate the real astrophysical conditions. These calculations fall into two categories: laminar calculations in conditions of very high viscosity, "convection in treacle", and large eddy simulations with some sub-grid scale modelling of the turbulent flow. Sub-grid modelling approximations is an area that requires much detailed study, Canuto (1996) has recently claimed that many schemes in current use are inconsistent as they do not satisfy Galilean invariance - this criticism (fortunately!) does not apply to the Smagorinski scheme used by myself and colleagues Kwing Chan and Harinder Singh.

3-D simulations by Nordlund and Stein (eg 1997) of the solar convective envelope are most impressive in that they include radiative losses at the upper boundary. Their results on overshooting at the base of the layer do not give a sharp transition between the convective region - which includes a large marginally stable zone - and the radiative interior, the sharp transition being smoothed out by averaging over many penetrat- 


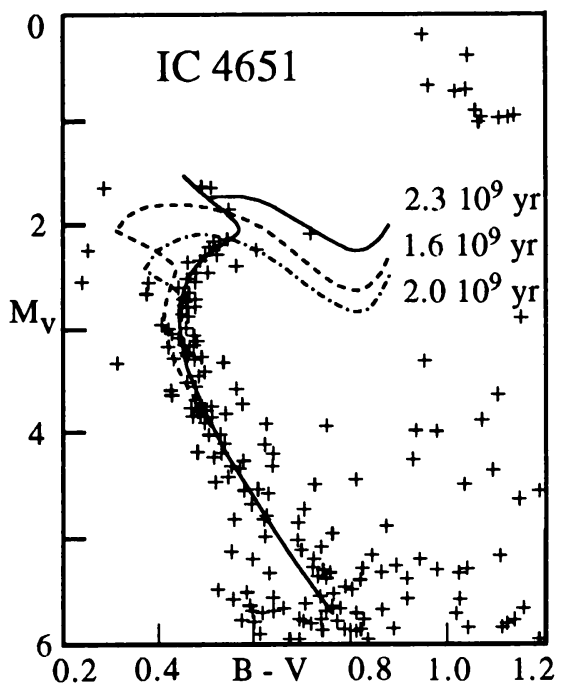

Figure 1. The best isochrone fit to Cluster IC 4651 is with core overshooting using the Integral Constraint, and $t=2.310^{9}$ yrs. Standard models give a worse fit and a younger age (Dowler and VandenBerg)

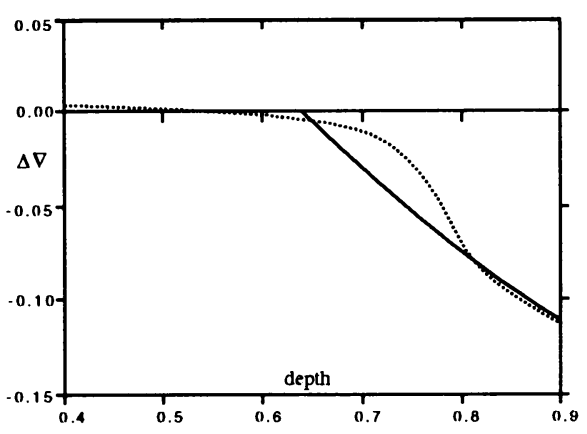

Figure 3. Variation of the superadiabatic gradient $\Delta \nabla$ at the boundary of a convective layer: numerical solution with $\sigma=0$ (dotted line) and mixing length model (solid line).

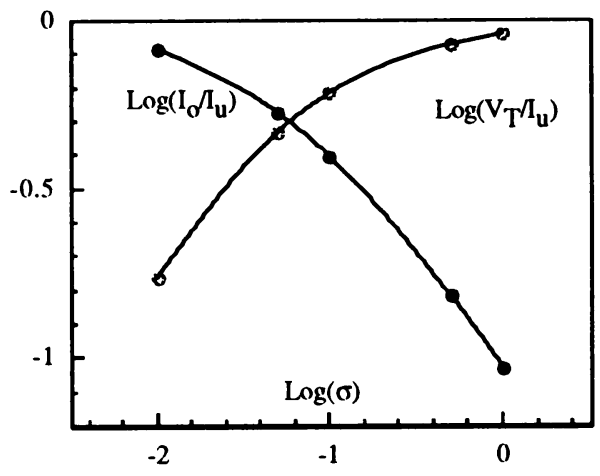

Figure 2. Variation with Prandtl number $\sigma$ of the ratio of the contributions to the integral constraint from the overshoot region $I_{o}$ and the total viscous dissipation $V_{T}$, to the contribution from the unstable region $I_{u}$

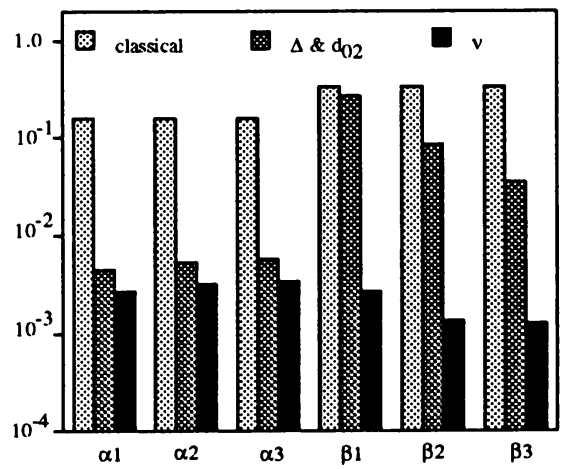

Figure 4. Error estimation on the determination of core overhooting parameters $\beta_{i}(M)$ and mixing length parameters $\alpha_{i}(M)$ for a set. of 6 stars in a model Hyades cluster. 
ing plumes. 2-D resolved laminar simulations by Roxburgh and Simmons (1993) do however find such a penetration layer that is at least similar to that predicted by simple analytical models (Figure 3). Likewise 3-D laminar simulations by Roxburgh (1998) and turbulent simulations by Singh, Roxburgh and Chan $(1994,95,97)$ using Smagorinski sub-grid modelling, find such a transition. Much remains to be done!

\section{Asteroseismology and convection}

A major advance in our understanding of convective cores should be achieved through asteroseismology, especially through high precision space observations such as those planned for the French satellite mission COROT (cf. Baglin et al. 1997), and possible larger missions such as STARS (Badiala et al. 1996). Advances should also be achieved through the development and application of diagnostic and inversion techniques to coordinated ground based observations of (for example) $\delta$ Scuti and $\beta$ Cephei stars. The boundary of convective cores generates a periodic signal in the oscillation frequencies produced by the steep change in sound speed gradients and composition as the star evolves with mixing in the core and associated overshoot region (Roxburgh and Vorontsov 1994). Some oscillation modes can be exceedingly sensitive to the region around the core, giving a valuable diagnostic tool with which to probe the internal core structure. The problem with such stars is that of mode identification and fitting the observed spectrum of frequencies to a model of the star. At the moment this is still an uncertain process but promises rich rewards. The method used is essentially model fitting, that is to produce a set of models of different mass and with different assumptions on the internal physics, in particular on convective overshooting, that satisfy such classical observational constraints as exist $\left(M_{V}, T_{\text {eff }}\right.$, abundances), computing the oscillation frequencies of these models and seeking to find a fit to the observations. This is not easy since only a subset of modes may be observed and because the predicted values of the frequencies depends on the structure the surface layers where there is considerable uncertainty in our understanding of the physics. A new "differential response" technique which gives a way round this problem has recently been developed by Vorontsov (1997) and gives some hope that progress can be made in this area.

For a group of stars in a cluster, or in a binary system, asteroseismology is a potentially powerful tool for probing both convective overshooting in the core and the properties of convection in outer envelopes. For such stars which may be assumed to have the same age and initial composition (and in the case of binaries possibly good constraints on the masses) we can parametrise the unknowns in the models of stellar evolution and seek to determine these by a simultaneous fit to the classical observables $\left(M_{V}, T_{\text {eff }}\right.$, and possibly some constraints on composition $\left.X, Y, Z\right)$ and the measured oscillation frequencies. Several such analyses have been undertaken to quantify the accuracy with which one can determine these parameters by such cluster/group fitting (cf. Audard et al. 1996). Figure 4 shows the results of an analysis by Audard and Roxburgh (1997) for a group of 6 stars with properties similar to those in the Hyades, in which the unknown stellar parameters are $\beta_{i}$, the fractional increase in core mass due to overshooting (assumed to vary with core mass), the parameter $\alpha_{i}$ in the mixing length model of convection, (assumed to vary with stellar surface properties $g, T_{\text {eff }}$ ), the masses of the stars $M_{i}$, the age $t$, the initial composition $Y, Z$, and the distance to the cluster $D$. The classical observables and their assumed errors are magnitudes $m_{V}(0.01 \mathrm{mag}), B-V(0.01 \mathrm{mag}), \log Z(0.1), \pi(0.004 ")$. The 
asteroseismological observables are assumed to be frequencies with $\ell=0,1,2$ either just enough to give an average value (over $n, \ell$ ) for the large separation $\Delta=\nu_{n, \ell}$ $\nu_{n-1, \ell}(0.05 \mu \mathrm{Hz})$ and small separation $d=\nu_{n, \ell}-\nu_{n-1, \ell+2}(0.3 \mu \mathrm{Hz})$, or individual frequencies $\nu_{n, \ell}(0.3 \mu \mathrm{Hz})$ with the assumption that for large mass stars only half the frequencies with $n=1,10$ are measured, and for smaller mass stars half the frequencies with $n=17,29$. This "cluster fitting" procedure needs to be refined both for binary systems and for clusters to demonstrate (with artificial data) that it is possible to reproduce the input physics that went into producing the artificial data, and in particular to use Vorontsov's "differential response" technique when individual frequencies are assumed measured.

\section{Conclusions}

Convection and convective overshooting is one of the most important and least understood processes that determine the structure and evolution of the sun and stars. There is evidence from observations of clusters and binary systems that penetration above convective cores is significant. A major advance can be expected from seismic observations of other stars, from the ground and from space, providing data that can be used to diagnose the properties of convective core overshooting and the efficiency of surface layer convection. Numerical simulations, whilst restricted in the range of parameters they can study, can also be used to address problems in this field and to guide theorteical developments. A major effort however needs to be directed towards understanding sub-grid scale modelling and eddy viscosity.

\section{References}

Audard et al. 1996, Bull. Astr. Soc. India, 24, 305

Audard N, Roxburgh I W, 1997, Proc IAU 181, ed F-X Schmider, in press

Badiala, M, et al., 1996. STARS. ESA D/ScI 96/4, Paris.

Balgin A et al., 1997 COROT, Convection and Rotation, Phase A study, CNES, France.

Canuto V., 1997, Solar Convection Oscillations and their Relationship, eds Pijpers F, Christensen-Dalsgaard J and Rosenthal C, Kluwer, Dordecht, 1997.

Dowler P, 1995, Thesis, University of Vancouver.

Dowler P., VandenBergh D., 1995, (private communication).

Maeder A, 1975, Astron. Astrophys, 40, 303

Maeder A, Mermilliod J-C,1992, Astron. Astrophys. Supp. 98, 477

Meynet G., Mermilliod J-C., Maeder A., 1993, Astron \& Astrophys Supp, 98, 477.

Nordlund $\AA$. Stein R.F., 1997, this volume

Roxburgh I W, 1965, Mon. Not. R. astr. Soc., 130, 223

Roxburgh I W, 1978, Astron. Astrophys 65, 281

Roxburgh I W, 1985, Solar Physics, 100, 21-51

Roxburgh I W, 1989, Astron. Astrophys 211, 361, 1992, ibid 266, 291

Roxburgh I W, 1998, to be published

Roxburgh I W, Simmons J., 1993, Astron. Astrophys, 277, 93

Roxburgh I W, Vorontsov S V., 1994, Mon. Not. R. astr. Soc., 267, 297

Schmitt J H M M, Rosner R, Bohm H U, 1984, Astrophys J., 282, 316.

Shaviv G and Salpeter E, 1973, Astrophys J., 184, 191

Singh H P, Roxburgh I W, Chan K L, 1994, Astron. Astrophys, 281, L73, 1995, ibid, 295, 703,1997 , this volume.

Vorontsov S V, 1997, Sounding Solar and Stellar Interiors, ed F-X Schmider, in press

Zahn J-P, 1991, Astron. Astrophys., 252, 179 